\title{
AMBER and CRIRES observations of the binary sgB[e] star HD 327083: evidence of a gaseous disc traced by $\mathrm{CO}$ bandhead emission ${ }^{\star}$
}

\author{
H. E. Wheelwright ${ }^{1}$, W. J. de Wit ${ }^{2}$, G. Weigelt ${ }^{1}$, R. D. Oudmaijer ${ }^{3}$, and J. D. Ilee ${ }^{3}$ \\ 1 Max-Planck-Institut für Radioastronomie, Auf dem Hügel 69, 53121 Bonn, Germany \\ e-mail: hwheelwright@mpifr-bonn.mpg.de \\ 2 European Southern Observatory, Alonso de Cordova 3107, Vitacura, Santiago, Chile \\ 3 School of Physics and Astronomy, University of Leeds, Leeds LS2 9JT, UK
}

Received 1 April 2012 / Accepted 29 May 2012

\begin{abstract}
Context. HD 327083 is a supergiant B[e] star that forms a binary system with an orbital semi-major axis of approximately 1.7 AU. Aims. Our previous observations using the VLTI and AMBER in the medium resolution $K$-band mode spatially resolved the environment of HD 327083. The continuum visibilities obtained indicate the presence of a circumbinary disc. CO bandhead emission was also observed. However, due to the limited spectral resolution of the previous observations, the kinematic structure of the emitting material could not be constrained. In this paper, we address this and probe the source of the $\mathrm{CO}$ emission with high spectral resolution and spatial precision.

Methods. To determine the properties and kinematics of its CO emitting region, we have observed HD 327083 with high spectral resolution (25 and $6 \mathrm{~km} \mathrm{~s}^{-1}$ ) using AMBER and CRIRES. The observations are compared to kinematical models to constrain the source of the emission.

Results. The multi-epoch AMBER spectra obtained over 5 months contain no evidence that the CO 1st overtone emission of HD 327083 is variable. This indicates that the structure of the emitting region is not strongly dependent on orbital phase. It is shown that the $\mathrm{CO}$ bandhead emission can be reproduced using a model of a Keplerian disc with an inclination and size consistent with our previous VLTI observations. The model is compared to AMBER differential phase measurements, which have a precision as high as $\sim 30 \mu$ as. A differential phase signal corresponding to 0.15 mas $(\sim 5 \sigma)$ is seen over the bandhead emission, which is in excellent agreement with the model that fits the CRIRES observations. In comparison, a model of an equatorial outflow, as envisaged in the standard $\mathrm{sgB}[\mathrm{e}]$ scenario, does not reproduce the observations well.

Conclusions. We present a direct test of the circumstellar kinematics of the binary sgB[e] star HD 327083 using both spatial and spectral information. The excellent agreement between the disc model and observations in the spatial and spectral domains is compelling evidence that the $\mathrm{CO}$ bandhead emission of HD 327083 originates in a circumbinary Keplerian disc. In contrast, the model of an equatorial outflow cannot reproduce the observations well. This suggests that the standard $\mathrm{sgB}$ [e] scenario is not applicable to HD 327083, which supports the hypothesis that the B[e] behaviour of HD 327083 is due to binarity.
\end{abstract}

Key words. circumstellar matter - stars: early-type - stars: emission-line, Be - stars: mass-loss - stars: individual: HD 327083

\section{Introduction}

Massive stars play a crucial role in multiple areas of astrophysics. As a result, a complete understanding of many astrophysical phenomena requires an understanding of the evolution of massive stars. In turn, this requires knowledge of how massive stars lose mass (see e.g. Puls et al. 2008). Supergiant B[e] $(\mathrm{sgB}[\mathrm{e}])$ stars are important objects in this regard as they are massive objects in a late evolutionary stage which also exhibit signs of enhanced mass loss (see e.g. Lamers et al. 1998). In addition, it has been suggested that there could be an evolutionary link between $\mathrm{sgB}[\mathrm{e}]$ stars and the luminous blue variables (LBVs, Zickgraf 2006). In turn, LBVs are generally thought to be the precursors of the Wolf-Rayet stars; the final phase in massive star evolution (see e.g. Vink 2009). Therefore, the asymmetric geometries that are required to produce phenomena such

* Based on data obtained at the European Southern Observatory, Paranal, Chile via the observing programmes 385.D-0513 and 087.D-0889. as gamma ray bursts may be constructed in the $\mathrm{sgB}[\mathrm{e}]$ stage. Consequently, it is important to study the circumstellar geometry of $\mathrm{sgB}[\mathrm{e}]$ stars and determine what is responsible for their mass loss.

With this in mind, we recently studied the $\operatorname{sgB}[\mathrm{e}]$ star HD 327083 with the VLTI and AMBER in the medium resolution $K$-band mode (see Wheelwright et al. 2012b, hereafter W12). High spatial resolution observations were required to probe the geometry of the object's circumstellar environment on milli-arcsecond scales. In turn, this was necessary to elucidate the "bracket" behaviour of HD 327083, for which there have been several hypotheses. On the basis of a comparison between optical spectroscopy and a NLTE model of an expanding atmosphere, Machado \& de Araújo (2003) suggest that the object may be close to the LBV phase. This would make it a key object to study the link, if any, between the $\mathrm{sgB}[\mathrm{e}]$ and LBVs. However, there is an alternative hypothesis. Miroshnichenko et al. (2003, hereafter M2003) detected an unresolved binary companion via radial velocity variations. Based on the relatively short period of 
Table 1. The log of the AMBER observations.

\begin{tabular}{lcccccc}
\hline \hline Date & Stations & $\begin{array}{c}\text { Seeing } \\
\left({ }^{\prime \prime}\right)\end{array}$ & $\begin{array}{c}\text { Coherence time } \\
(\mathrm{ms})\end{array}$ & $\begin{array}{c}\text { DIT } \\
(\mathrm{s})\end{array}$ & $\begin{array}{c}\text { Baseline } \\
(\mathrm{m})\end{array}$ & $\begin{array}{c}\text { PA } \\
\left({ }^{\circ}\right)\end{array}$ \\
\hline 02-05-2011 & H0-I1-G1 & 0.46 & 5.3 & 12.0 & $40.7 / 38.6 / 65.7$ & $169.9 / 58.1 / 23.0$ \\
07-05-2011 & D0-A1-C1 & 0.68 & 4.1 & 12.0 & $34.7 / 15.7 / 21.8$ & $45.4 / 71.7 / 26.9$ \\
12-06-2011 & D0-B2-C1 & 0.88 & 4.6 & 12.0 & $33.2 / 11.0 / 22.1$ & $22.2 / 22.1 / 22.2$ \\
27-07-2011 & K0-I1-G1 & 1.03 & 2.1 & 12.0 & $44.2 / 43.6 / 84.9$ & $19.3 / 49.0 / 34.0$ \\
04-08-2011 & K0-I1-G1 & 0.74 & 2.1 & 12.0 & $45.1 / 45.9 / 88.1$ & $11.1 / 39.5 / 25.4$ \\
22-09-2011 & K0-I1-G1 & 0.77 & 3.1 & 12.0 & $38.2 / 29.5 / 65.1$ & $33.9 / 66.2 / 47.9$ \\
\hline
\end{tabular}

Notes. The baseline lengths and position angles (PAs) represent time-averaged values for a given night.

the variations, $\sim 6$ months, these authors suggest that the system is close enough to interact. In this case, the material traced by the object's infrared excess may be mass lost as the result of binary interactions, as proposed for other objects showing the $\mathrm{B}[\mathrm{e}]$ phenomenon (see Millour et al. 2009, 2011; Kraus et al. 2012).

We now briefly summarise the results from W12. Our observations with the VLTI and AMBER spatially resolved the material responsible for the $K$-band excess of HD 327083. Using simple geometrical models, we found that the continuum visibilities could be reproduced by an elongated ring with a central radius of approximately 6.6 AU. The location of this ring is consistent with the expected dust sublimation radius of a star with the stellar parameters reported by M2003; $6<R_{\text {sub }}<25$ AU using the standard equation (see Monnier \& Millan-Gabet 2002). A nonzero closure phase was observed over the continuum emission and this was attributed to the binary companion. The closure phase suggested that the companion should be located within the elongated ring. Therefore, the geometrical model was associated with the inner rim of a dusty, circumbinary disc. Such a configuration is more reminiscent of mass lost as the result of binary interactions (see e.g. Millour et al. 2011) than intrinsic mass loss via an equatorial outflow, as depicted in the standard $\mathrm{sgB}[\mathrm{e}]$ scenario (see e.g. Zickgraf et al. 1985). Therefore, the observations to date favour the interacting binary hypothesis.

Probing the mass loss process of the system further requires constraining the kinematics of the circumbinary material and the conditions that give rise to the occurrence of the $\mathrm{B}[\mathrm{e}]$ phenomenon. Such constraints can be determined from spectrally resolved observations of $\mathrm{CO}$ bandhead emission. Therefore, we obtained new high spectral resolution $\left(25\right.$ and $\left.6 \mathrm{~km} \mathrm{~s}^{-1}\right)$ observations of HD 327083's CO bandhead emission with AMBER and CRIRES. Here we present these observations, which provide sub-milli-arcsecond precision constraints on the geometry of the emitting material. The CO bandhead emission of HD 327083, initially observed by McGregor et al. (1988), is spectrally resolved for the first time and we observe a differential phase signature over the emission. This paper presents the observations and modelling of the $\mathrm{CO}$ emitting material, and it is structured as follows. In Sect. 2 we describe the observations and subsequent data reduction. We present the observational results in Sect. 3. These are then analysed and modelled in Sect. 4 and the implications are discussed in Sect. 5. The conclusions are presented in Sect. 6.

\section{Observations and data reduction}

\subsection{CRIRES}

HD 327083 was observed with the near-IR cryogenic high resolution spectrograph CRIRES of the VLT (Kaeufl et al. 2004) during the night of June 28, 2010. The target was included in our survey of sixteen Galactic, $K$-band bright $\operatorname{sgB}[\mathrm{e}]$ candidates

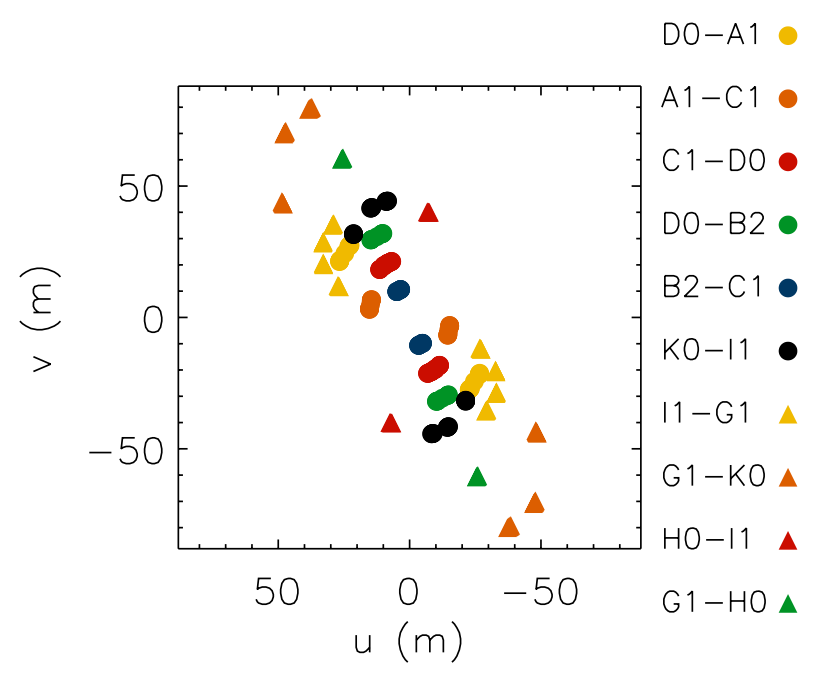

Fig. 1. The projected baselines of the AMBER observations of HD 327083.

and unclassified $\mathrm{B}[\mathrm{e}]$ stars. The aim of the project is to study in detail the kinematic signature of the $\mathrm{CO}$ first overtone bandhead of $\mathrm{B}[\mathrm{e}]$ stars in general (Muratore et al., in prep.). Here we make use of the data for this object. Observations were executed with a slit-width of $0.4^{\prime \prime}$ delivering a spectral resolution of approximately 50000 (or $6 \mathrm{~km} \mathrm{~s}^{-1}$ ). The data were obtained applying a standard nodding on-slit strategy to remove sky and detector glow, without AO correction and applying a small jittering offset at each nod position to better remove bad pixels. The DIT was chosen bearing in mind the non-linearity regime of the CRIRES InSb Aladdin III detectors. A telluric standard was taken immediately after the science targets, close in airmass. The seeing conditions were poor as the seeing varied between 1.75 and $2.25^{\prime \prime}$. However, the brightness of the target, $K=3.3 \mathrm{mag}$, ensured the data are still of high quality. Data reduction was conducted in a standard fashion utilising the ESO provided CRIRES pipeline.

\section{2. $A M B E R$}

HD 327083 was observed with the VLTI and the 3-beam combiner instrument AMBER (Petrov et al. 2007) in the high spectral resolution $K$-band mode centred on $2.288 \mu \mathrm{m}$. This setting delivers a spectral resolution of $R=12000$ and a wavelength range of 2.265 to $2.311 \mu \mathrm{m}$. FINITO was used to provide fringe tracking. Observations were conducted on 6 occasions between May 2nd 2011 and September 22nd 2011. The observations were carried out employing the $1.8 \mathrm{~m}$ Auxiliary Telescopes (ATs). A $\log$ of the observations is presented in Table 1 and the projected baselines are presented in Fig. 1. In general, short baselines were 
used as our previous observations demonstrate that this object is relatively extended. The seeing conditions were typically good (the mean seeing was $\sim 0.75^{\prime \prime}$ ), which was important to facilitate these high spectral resolution observations. Observations of HD 327083 took place between observations of calibrator objects (HD 151078 and HD 161068).

The AMBER observations were reduced in a standard fashion using the amdlib software version 3.0.3 (see Tatulli et al. 2007; Chelli et al. 2009). The processes involved, i.e. determining the pixel-to-visibility-matrix and converting the observed fringe patterns to measurements of the coherent flux, are standard operations with this software and are not described here. Once the interferometric observables are calculated, the general approach is to subject each block of frames to certain selection criteria before averaging the selected frames into a single measurement. Since we employed the high spectral resolution mode of AMBER with a long integration, and the data exhibit a range of frame signal-to-noise-ratios, it is challenging to accurately calibrate the visibilities. Therefore, we do not make use of absolute visibilities in this paper. As we are not concerned with temporal variations in the transfer function, we merge all consecutive observations before performing frame selection and averaging. This provides a higher resultant signal-to-noise-ratio (SNR) (see e.g. Štefl et al. 2009). A high frame selection rate, 80 per cent, was used as this generally provides the highest quality phase information and our analysis in this paper concentrates on these measurements.

Wavelength calibration of the AMBER data was conducted using the telluric lines present in the spectra. To provide a reference wavelength for the telluric lines observed, we utilised a high resolution $(R=40000)$ spectrum of the Earth's telluric features made available by the NSO/Kitt Peak Observatory ${ }^{1}$. The position of the telluric lines was fit with a 2 nd order polynomial which was used to provide the wavelength calibration. The mean rms of the solutions was found to be approximately $4 \mathrm{~km} \mathrm{~s}^{-1}$, which was deemed acceptable. Only the spectra of HD 327083 were used to provide wavelength calibration as the spectra of the standards exhibit $\mathrm{CO}$ absorption features, which made identification of the telluric lines less secure.

On several occasions, the closure phases of the calibrator objects were non-zero. In an attempt to correct this, we subtracted the mean of the calibrator closure phases from those of HD 327083. Since we merged the OI files prior to frame selection and averaging, the closure phase data calibration could be compromised by temporal variations in the instrumental closure phase. In general, the closure phases of the two calibrators are consistent, indicating this is not an issue. However, the calibrator closure phases for the observations conducted in September were inconsistent by approximately $5^{\circ}$. This translates to a systematic uncertainty in the final calibrated measurement.

The two calibrator stars, HD 151078 and HD 161068, were also used as telluric standards. For each night of observing, we attempted to remove the telluric absorption features in the spectrum of HD 327083. Before this could be done, the $\mathrm{CO}$ absorption features in the spectra of the standard had to be removed (see e.g. W12, Tatulli et al. 2008). This was done by dividing the standard spectra with a telluric corrected spectrum of a template star with the same spectral type. High-resolution $(R \sim 18000), K$-band template spectra were obtained from the library of 25 standard stars observed with GNIRS provided by

\footnotetext{
1 http://www . eso.org/sci/facilities/paranal/ instruments/isaac/tools/spectroscopic_standards.html\# Telluric
}

GEMINI observatory ${ }^{2}$. For HD $161058\left(\mathrm{~K} 4 / \mathrm{K} \mathrm{III}^{3}\right)$ the spectrum of HD 78541, also of spectral type K4/5III was used. In the case of HD 151078 (K0III) the average spectrum of HD 74137 and HD 28 (both K0III) was used.

\section{Observational results}

The AMBER and CRIRES observations spectrally resolve the CO bandhead emission of HD 327083 for the first time (the spectra are presented in Sects. 4.1 and 4.2 respectively). The high spectral resolution of CRIRES $\left(\sim 6 \mathrm{~km} \mathrm{~s}^{-1}\right)$ and the high SNR of the data $(\sim 200)$ provide a unique view of the bandhead spectral profile. The bandhead clearly displays a blue wing, the so-called "blue shoulder", which can indicate that the emission originates in a rotating medium such as a disc (see e.g. Wheelwright et al. 2010, hereafter W10, and references therein). No clear signature is seen over the bandhead emission in the differential visibilities. However, we report the first detection of a signal across $\mathrm{CO}$ bandhead emission in AMBER differential phase observations (this is presented in Sect. 4.3). This allows us to probe the emitting region with a precision as high as $30 \mu$ mas.

Turning to the closure phase measurements (presented in Sect. 4.4), no clear signature is seen over the CO bandhead emission. In general, the average closure phases are close to zero; which suggests that the environment of HD 327083 is relatively symmetric. However, the observations in July suggest a shift to non-zero values, which indicates an increase in the asymmetry of the environment of HD 327083. In the following sections we analyse these points in more detail to constrain the source of the CO emission and the nature of the mass loss of HD 327083.

\section{Analysis and modelling}

\subsection{CO bandhead spectra: assessment of variability}

Our AMBER observations of HD 327083 span a period of approximately 5 months, which is more than 80 per cent of the orbital period estimated by M2003. Therefore, we can use the observed flux spectra to assess whether the $\mathrm{CO}$ emission is variable, which might indicate mass transfer at particular orbital phases. To determine whether there are significant differences between the average spectra from each night of observation, we use the temporal variance spectrum (TVS, see Fullerton 1990). The TVS can be expressed by:

$(T V S)_{\lambda} \approx \frac{1}{N-1} \sum_{i=1}^{N}\left[\frac{F_{i}(\lambda)-F_{\mathrm{av}}(\lambda)}{\sigma_{i}(\lambda)}\right]^{2}$

where $N$ represents the number of spectra, $F_{i}(\lambda)$ denotes the individual spectra, $F_{\text {av }}(\lambda)$ is the average spectrum and $\sigma_{i}$ is the uncertainty at each point of the individual spectra. Here, we used the rms in the continuum as a constant uncertainty across the individual spectra.

Following the example of Oudmaijer \& Bakker (1994), we calculated the temporal sigma spectrum, TSS, which is given by $T S S=\sqrt{T V S}$. This value is expressed in terms of the noise level. Therefore, if there are no significant deviations between the individual spectra, this value will be approximately unity. On the other hand, significant variations will be associated with a large TSS value. The average AMBER spectrum of HD 327083

\footnotetext{
2 http://www.gemini.edu/sciops/instruments/ nearir-resources/spectral-templates/r18000/

3 From SIMBAD: http://simbad.u-strasbg.fr/simbad/
} 


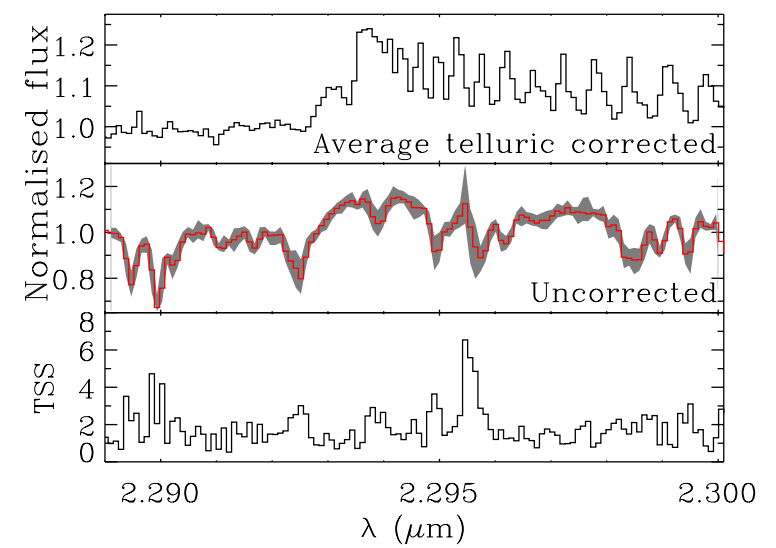

Fig. 2. The upper panel presents the time-averaged, telluric corrected AMBER spectrum. The central panel contains the un-processed (not corrected for telluric absorption) AMBER spectrum. The shaded region represents the scatter of the six average spectra constructed for each observing date. The lower panel presents the temporal sigma spectrum.

(uncorrected for telluric absorption) and the associated TSS is shown in Fig. 2. HD 327083 was observed at different air-masses each of the six times it was observed. As a result, the strength of the telluric absorption features in each spectra is different, although, as shown in Fig. 2, the differences are relatively small. The telluric lines in the average spectrum are associated with peaks in the TSS. This confirms that this approach is sensitive to variability. The bandhead itself, however, exhibits no such feature. Therefore, we conclude that these data contain no evidence of variability in the $\mathrm{CO}$ bandhead emission.

We note that the final telluric corrected AMBER and CRIRES spectra are essentially consistent, which is to be expected if the emission is not variable. However, comparison of the two suggests that the telluric correction in the AMBER spectrum is not perfect. This is likely because the standards were chosen as interferometric calibrators rather than telluric standards and thus they do not necessarily provide the best air-mass match. From here-on, as far as flux spectra are concerned, we concentrate on the high spectral resolution CRIRES spectrum.

\subsection{Modelling the CO flux spectrum}

With the aim of constraining the origin and kinematics of the bandhead emission, the observed spectral profile is fit with a kinematical model. Specifically, to evaluate the disc scenario, we attempt to fit the $\mathrm{CO}$ bandhead with a model of a rotating disc. We have already used a similar model to fit the $\mathrm{CO}$ emission of massive young stellar objects in other publications (W10, Ilee et al., in prep.), and we thus only describe the methodology briefly here.

A simple, geometrically flat disc in Keplerian rotation is constructed. The excitation temperature and surface number density decrease with increasing radius according to power laws. The temperature decreases with $R^{-0.75}$ and the surface number density falls off with $R^{-1.5}$, in line with standard disc theory (see e.g. Kraus et al. 2000). We note that the temperature is the excitation temperature used to determine the relative populations of the CO ro-vibrational energy levels. Therefore, it may not necessarily follow the thermal temperature distribution. For example, it is possible the vibrational levels are not in LTE (Najita et al. 1996; Martin 1997). The excitation temperature distribution is difficult to constrain with only one bandhead profile as it can
Table 2. The parameters of the $\mathrm{CO}$ emitting Keplerian disc model that best fits the CRIRES spectrum.

\begin{tabular}{lcc}
\hline \hline Parameter & Value & Notes \\
\hline Cent. Mass & $25 M_{\odot}$ & Set $^{1}$ \\
$R_{\star}$ & $25 R_{\odot}$ & Set $^{2}$ \\
$i$ & 45.8 & Set $^{3}$ \\
$N_{0}$ & $0.4 \pm 1.8 \times 10^{24} \mathrm{~cm}^{-2}$ & \\
$R_{\text {in }}$ & $25.5 \pm 2.3 R_{\star}$ & $\sim 3 \mathrm{AU}, 2$ mas \\
Linewidth & $3.2 \pm 3.2 \mathrm{~km} \mathrm{~s}^{-1}$ & \\
$T_{0}$ & $1721 \pm 540 \mathrm{~K}$ & \\
$\chi^{2}$ & 3.96 & \\
\hline
\end{tabular}

Notes. 1) Based on an early B type primary and an F type companion (M2003); 2) this sets the minimum possible inner disc radius, based on the values reported by M2003; 3) set by our previous AMBER observations (W12) and assuming that the CO emission is co-planar with the continuum emission. The outer radius is simply the radius at which the temperature falls below $1000 \mathrm{~K}$, and in this case is approximately $6 \mathrm{AU}$.

vary significantly (see e.g. Berthoud et al. 2007). Therefore, we fix the temperature distribution as described.

The temperature and surface density at the inner radius, $R_{\text {in }}$, are free parameters. The $\mathrm{CO}$ emission is assumed to originate in the region of the disc where $1000 \mathrm{~K} \leq T \leq 5000 \mathrm{~K}$. In the case of HD 327083, we set the inclination of the disc to be $48.5^{\circ}$, as determined by W12. In most cases, it would be assumed that the disc extends from the stellar surface. However, in the case of HD 327083, it is possible that the disc is circumbinary and the inner disc is cleared. Therefore, we allow the inner rim of the disc to vary. The free parameters of this particular model are thus: the inner rim of the disc (technically the radial distance of the smallest annulus), the temperature at $R_{\text {in }}$, the intrinsic line-width and the surface number density at $R_{\text {in }}$. The disc was split into radial and azimuthal cells and the $\mathrm{CO}$ emission of each cell was calculated according to the methodology of Kraus et al. (2000). Finally, once the individual cell spectra were calculated, they were summed to create the total spectrum. This was then smoothed to match the spectral resolution of the observations.

This relatively simple approach is standard in such studies as there are no sophisticated models including $\mathrm{CO}$ bandhead emission available. As the code is analytic, it is readily used in a fitting scheme. The best-fitting model was determined using the downhill simplex algorithm (implemented in IDL as the amoeba routine). Once the best fit model was found, monochromatic images were created, which were subsequently compared to the interferometric data. The uncertainties associated with the final parameters were determined in the following way. A 2D reduced $\chi^{2}$ map was constructed for each set of parameters, centred on the best fitting model. The uncertainties in the best fitting parameters were estimated by determining the maximum parameter change that results in a change in $\chi^{2}$ of 1 . As discussed in Domiciano de Souza et al. (2011), this likely results in an over-estimation of the uncertainty (provided the data points are un-correlated). To investigate the possibility of additional fits with different inner radii, we constructed 2D $\chi^{2}$ maps centred on the best fitting model but displaced in the direction of the inner radius by -5 and $+5 R_{\star}$ (we concentrated on the inner radius as this influences the interpretation of the data, as discussed in Sect. 5). In both cases, the minimum $\chi^{2}$ was found to be greater than that of the model presented in Table 2. Therefore, we conclude that the best fitting model does indeed correspond to the global $\chi^{2}$ minimum. 

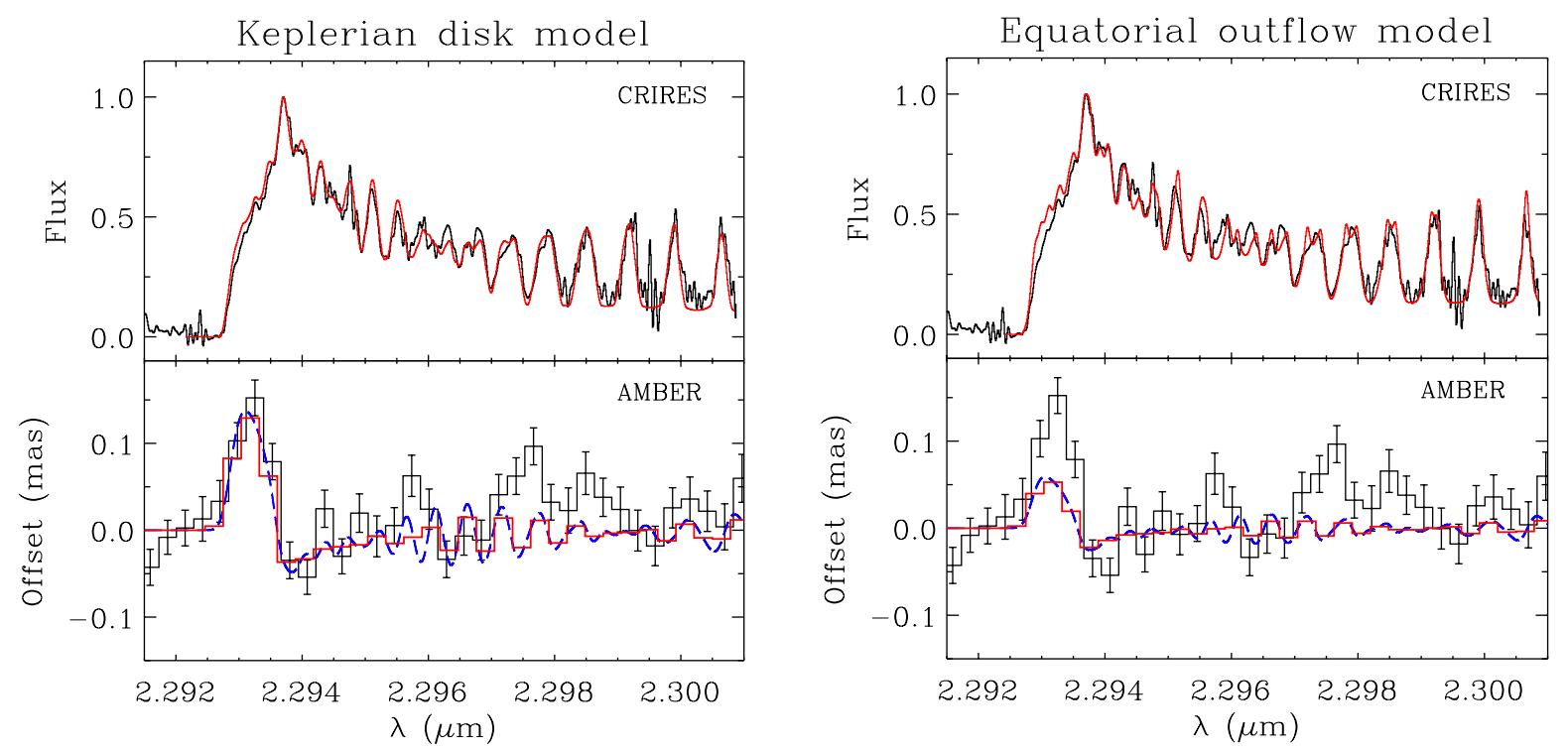

Fig. 3. A comparison between the observations and the two physically different best fitting models explored in the text. The upper panels present the average CRIRES spectrum and the profile of the best fitting model. In the lower panels, the observed photo-centre offsets calculated using AMBER differential phase measurements from the baseline with the lowest noise level (D0-A1 used on the 7th of May) are shown. For each model that fits the CRIRES spectrum we over-plot the predicted signature for the selected baseline. The model signatures are smoothed to the spectral resolution of AMBER and are shown re-binned using the size of the AMBER pixels and with a finer sampling.

The model spectrum that is found to best reproduce the $\mathrm{CO}$ bandhead profile is shown in the top panel of Fig. 3. The parameters of the best fitting model and the adopted stellar parameters are presented in Table 2. In general, the model provides an excellent match to the features of the bandhead. Indeed, the fit is remarkably good considering the limited number of free parameters and the simplicity of the model. Therefore, we conclude that a model of a Keplerian disc with an inner radius of approximately $3 \mathrm{AU}$ and a relatively low initial temperature, $T_{0} \sim 1700 \mathrm{~K}$, appears to be a fitting representation of the $\mathrm{CO}$ emitting region associated with HD 327083.

However, the possibility remains that the source of the $\mathrm{CO}$ emission is not rotationally dominated. Indeed, the favoured model of the circumstellar environment of $\operatorname{sgB}[\mathrm{e}]$ stars features an equatorial outflow. To investigate whether this scenario is applicable to HD 327083, we perform an additional fit to the CRIRES spectrum with an outflow model.

The standard model for the $\operatorname{sgB}[\mathrm{e}]$ environment is a dense equatorial outflow and a faster wind concentrated in the polar regions. The equatorial outflow is thought to be the result of rapid rotation and the bi-stability jump leading to enhanced line driven mass loss in the equatorial region (see e.g. Pelupessy et al. 2000). In modelling the emission from an equatorial outflow, we adopt the analytical treatment of Porter (2003) who compared the SEDs of disc and outflow models to observations of the $\mathrm{sgB}[\mathrm{e}] \mathrm{star} \mathrm{R} 126$. Specifically, the $\mathrm{CO}$ emission is modelled as originating in a flat structure containing both rotation and outflow. The velocities are given by:

$v_{\mathrm{Rot}}=\sqrt{G M_{\star} / R_{\star}}\left(\frac{r}{R_{\star}}\right)^{-1}$

and

$v_{\text {Out }}=v_{\text {Sound }}+\left(v_{\infty}-v_{\text {Sound }}\right)\left(1-\frac{R_{\star}}{r}\right)^{\beta}$

which represent angular momentum conserving rotation and the velocity structure of a line driven wind respectively.
We fit the CRIRES spectrum with a geometrically flat, equatorial outflow model featuring both these velocity laws by treating the terminal velocity, line-width, initial number density and temperature as free parameters and setting $R_{\text {in }}=1 R_{\star}$ (since the wind is launched from the star) and $\beta=0.8$ (which is appropriate for a hot star wind). The inclination was also set, as in the disc modelling. The temperature and number density distributions follow the same power laws as in the disc model, which is appropriate for this analytic treatment (see e.g. the discussion on the heating of circumstellar discs and winds in Porter 2003). As before, the best fitting model was found using the downhill simplex algorithm.

The best fitting spectral profile of the equatorial outflow model is also shown in the upper panel of Fig. 3. The best fitting parameters are: $N_{0}=2.7 \times 10^{22} \mathrm{~cm}^{-2}, T_{0}=19624 \mathrm{~K}$, Linewidth $=6.8 \mathrm{~km} \mathrm{~s}^{-1}$ and $V_{\infty}=69.4 \mathrm{~km} \mathrm{~s}^{-1}$. As can be seen, the outflow model can approximately reproduce the CRIRES spectrum, although the quality of the fit is noticeably worse than that provided by the disc model. This is reflected in the large reduced $\chi^{2}$, which is $\sim 100$. Therefore, the $\chi^{2}$ values favour the Keplerian model. Nonetheless, since the spectrum is approximately recreated, it is not possible to confidently discard the outflow model based on the $\mathrm{CO}$ bandhead profile alone. However, the addition of our AMBER data provides an opportunity to differentiate between the two models. This is described in the following section.

\subsection{Differential visibilities and phases}

The spectral modelling of the previous section provides detailed predictions regarding the spatial distribution of the $\mathrm{CO}$ emission. Therefore, the AMBER data offer a unique test of the spectral models that fit the $\mathrm{CO}$ bandhead. No clear signature is seen in the differential visibilities across the bandhead emission. Considering the uncertainties in the visibilities (typically 10 per cent) and the low line-to-continuum flux ratio $(\sim 0.2)$, this is consistent with the $\mathrm{CO}$ bandhead emission originating close to, and perhaps interior to, the continuum emission (see the 

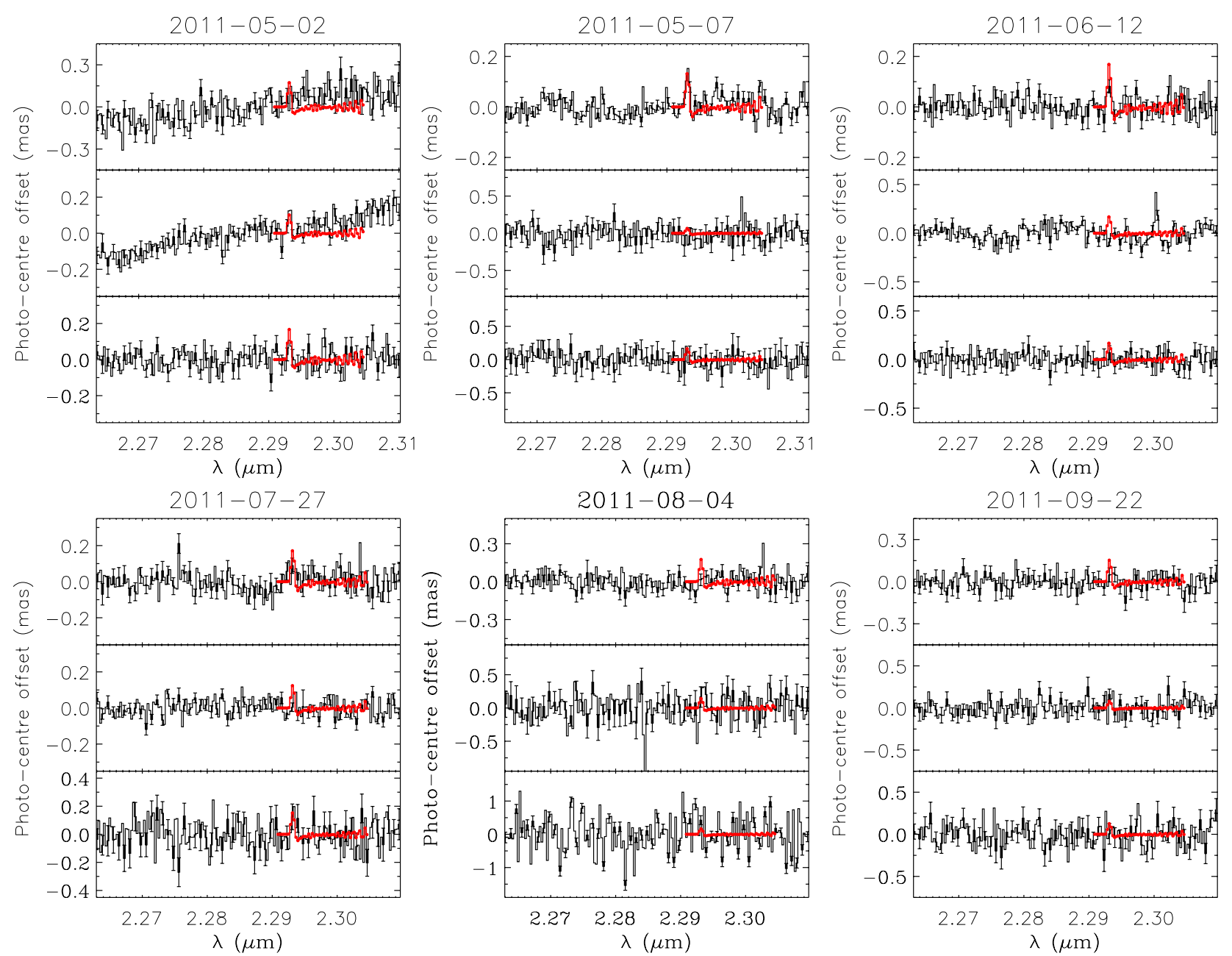

Fig. 4. Average differential phases converted into photo-centre shifts for each night of observing. The predictions of the disc model that fits the CRIRES spectrum are over-plotted.

discussion in W12). Our spectral models place the CO material interior to, but extending to, the continuum emitting region. Therefore, the best fitting models are consistent with the observed visibilities.

A second, more stringent test is provided by the AMBER differential phase measurements. Differential phases are sensitive to photo-centre displacements associated with asymmetric sources of flux, such as rotating discs (see e.g. Lachaume 2003). Therefore, we compare the differential phase measurements to the predictions of the models that fit the CRIRES data. To calculate the photo-centre offsets of the models, it is assumed that the continuum is centro-symmetric. Most of the closure phases measured are close to zero (see Sect. 4.4), indicating that the environment of HD 327083 is relatively symmetric, justifying this approach.

To improve the SNR of the data, we re-bin the differential phases by a factor of 3 (approximately a resolution element). The minimum uncertainty was $\sim 30 \mu$ as. This uncertainty corresponds to the data obtained with the $34.7 \mathrm{~m} \mathrm{D} 0-\mathrm{A} 1$ baseline used on 7 th May 2011. These data alone are precise enough to test the models at a significant level. The errors on the other baselines are generally a factor of two or more greater, so we concentrate on the data obtained with this baseline only.

A comparison between the predicted photo-centre offsets and that calculated from the differential phase measurements using the aforementioned baseline is presented in the lower panel of Fig. 3. The offsets calculated from the differential phases across the other baselines are presented in Fig. 4. The combination of high spatial and spectral precision provides stringent constraints on the kinematics of emission line regions. As can be seen, the predicted offsets of the best fitting disc and outflow models are different and thus we can use the spatial information provided by the differential phases to differentiate between the competing models.

We focus on the best fitting disc model first. As can be seen in Fig. 3, the maximum predicted offset occurs over the bandhead shoulder where many individual ro-vibrational lines are super-imposed (as discussed in W10). The offsets over the individual ro-vibrational lines are clearly too small $(<0.1$ mas) to be detected here. However, the predicted offset over the bandhead shoulder, $\sim 0.15$ mas, is large enough to detect with the current data, which has an noise level of $\sim 30 \mu$ as. The observations display a displacement of approximately 0.15 mas over the bandhead shoulder, in excellent agreement with the prediction of the model that fits the CRIRES spectrum. The significance of the peak pixel of the observed signature is approximately $5 \sigma$. The agreement between the amplitude of the model and observed signatures was optimised by slightly changing the position angle of the disc (by $10^{\circ}$ so that the difference between the position angle of the major axis of the disc and the baseline was $\sim 42^{\circ}$ ). This slight difference in PA could represent uncertainty in the previous estimate. However, it should be noted that the distance to HD 327083 has an uncertainty of approximately 30 per cent, which translates to a similar uncertainty in the model 

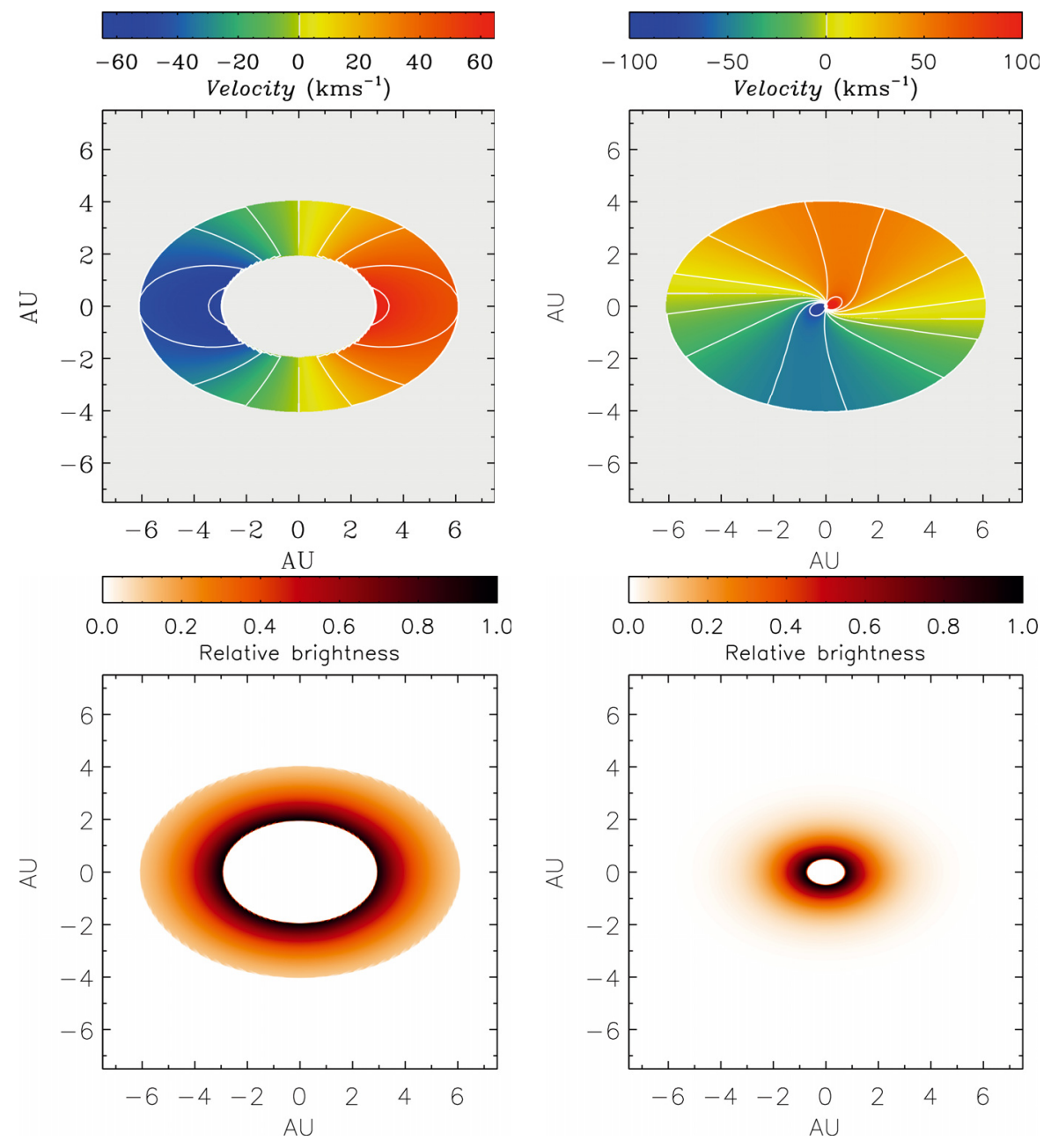

Fig. 5. A comparison between the velocity structure (upper row) and brightness distribution (lower row) of the best fitting disc (left column) and equatorial outflow (right column) models. The contours in disc velocity image are drawn at: $15,30,45$ and $60 \mathrm{~km} \mathrm{~s}^{-1}$ and the contour levels of the outflow velocity image are: $10,30,50$ and $70 \mathrm{~km} \mathrm{~s}^{-1}$. The maximum velocity shown in the outflow image is $100 \mathrm{~km} \mathrm{~s}^{-1}$, but the velocity at the inner edge of the structure is $\sim 400 \mathrm{~km} \mathrm{~s}^{-1}$. The brightness distributions are normalised to the contribution of the brightest element.

photocentre shift. Therefore, the agreement between the model and observations could also be optimised by slightly reducing the distance to HD 327083. As a result, the minor change in $\mathrm{PA}$ is not considered significant.

We emphasise that bar a modest adjustment of the PA, the observed differential phase signature was not fit. The model signature was predicted by the fit to the CRIRES spectrum. The excellent agreement between the model and the data in both the spectral and spatial domains strongly suggests the model is an accurate representation of the CO emitting region of HD 327083. We note that if the profile were fit with rotation that decreased more rapidly with radius than the Keplerian case, for example angular momentum conserving rotation in which $v \propto r^{-1}$, the resultant positional offset would be smaller than that observed and vice versa (see e.g. Wheelwright et al. 2012a). Therefore, the agreement between the model and data is compelling evidence that the $\mathrm{CO}$ bandhead emission traces a Keplerian disc.

Unlike the disc model, the outflow model cannot reproduce the data well. As shown, the photo-centre offset associated with the best fitting outflow model is smaller than that of the best fitting disc model. This is because the velocity structure and brightness distribution of the two best fitting models are very different. This is shown in Fig. 5. The $\mathrm{CO}$ emitting region of the outflow is clearly smaller than in the disc case. As a result, the spatial signature of the outflow is smaller than that of the disc and does not reproduce the differential phase shift seen (see Fig. 3). The discrepancy between the model and observations is too large to be accounted for by a modest change in PA or distance. We conclude that the large $\chi^{2}$ of the spectral fit and the poor reproduction of the photo-centre offset imply that the source of the $\mathrm{CO}$ emission is not an equatorial outflow and that these data favour the disc scenario.

\subsection{Closure phases}

The AMBER closure phase signal, sampled at different phases of the binary period ( $\sim 6$ months, Miroshnichenko et al. 2003), has the potential to trace changes in the degree of symmetry in the environment of HD 327083. This might be expected due to changes in the binary separation and PA and would allow us to constrain the binary orbit to a much higher degree. The wavelength-averaged closure phase measurements are presented in Fig. 6. All bar one of the closure phase measurements indicate, or are consistent with, a symmetric environment. Therefore, we do not definitively detect the binary companion. To investigate the geometrical constraints supplied by the closure phase observations, we fit the observations with two geometric models: that of a binary and the skewed ring model of Monnier et al. (2006). The binary model has only two free parameters, the binary PA and separation. The two stellar components are treated as unresolved uniform discs and the binary flux ratio was set to 3.5 , as discussed in W12. The parameters of the skewed ring model are: the ring radius and elongation, the ring flux, the ring PA, the degree of skewness and the PA of the skewed distribution. The properties such as the ring radius, inclination and flux were set to those of the ring found by W12 to represent the continuum emission of this object. Therefore our skewed ring model also has 2 unknowns: the degree of skewness and the PA of the skew. 

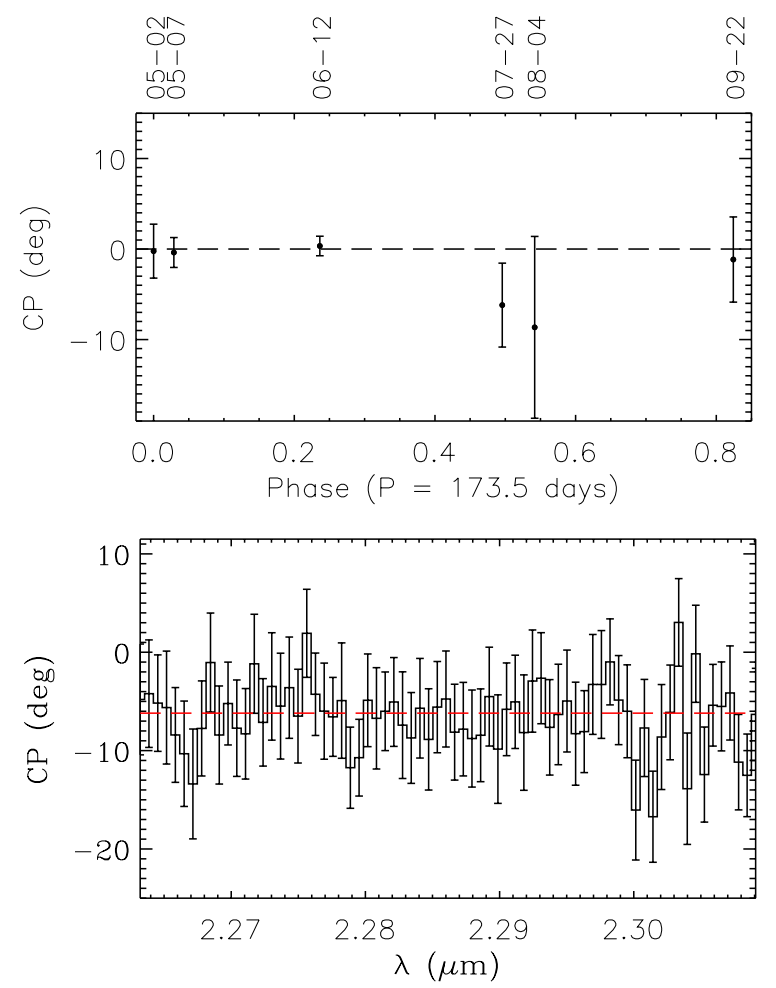

Fig. 6. The wavelength-averaged calibrated closure phase for each observing date (top panel) and an example of the closure phase data (data obtained on the 27 th of June, lower panel). The data have been rebinned by approximately twice the resolution element.

It was found that neither model could be excluded. Ultimately, the observations hint at an increase and subsequent decrease in the asymmetry of the environment of HD 327083. If this represents a change in the amount of asymmetry in the structure resolved in W12, its degree of asymmetry must increase on a timescale of several months. This appears unlikely given the size of the structure (approximately $5 \mathrm{AU}$ ). In addition, we note that the $\mathrm{CO}$ bandhead emission is located close to and interior to the continuum emission. Therefore, if the continuum emission varied, perhaps as a result of intermittent mass loss or accretion, variability in the $\mathrm{CO}$ emission would be expected. Since the $\mathrm{CO}$ emission is not variable, it appears that the continuum emission originates in a stable structure. Consequently, we attribute our tentative detection of a change in closure phase to motion of the binary system. A conclusive confirmation of the interferometric binary detection may require model independent imaging with, for example, the PIONIER instrument (e.g. as demonstrated by Blind et al. 2011).

\section{Discussion}

This paper presents high spectral resolution (25 and $6 \mathrm{~km} \mathrm{~s}^{-1}$ ) AMBER and CRIRES observations of the binary supergiant $\mathrm{B}[\mathrm{e}]$ star HD 327083. The combination of information on AU sized scales provided by the AMBER data and the velocity resolved information provided by the CRIRES data offers new insights into the close environment of this object. In particular, we show that the data can be reproduced well with a Keplerian disc model. On the contrary, the scenario of an equatorial outflow appears unlikely since such a model cannot reproduce the observations.

These observations, and the disc model that best fits the CRIRES spectrum, confirm several previous suggestions that were based on spectrally unresolved interferometric observations. In W12 it was remarked that there was no clear visibility signature across the $\mathrm{CO}$ overtone emission. On this basis, it was suggested that the $\mathrm{CO}$ emission originates from a region with the same elongation, and thus inclination, as the near-IR continuum. Furthermore, in W12 it was suggested that the CO material is located interior to the continuum emission. The geometry of the CO bandhead emission predicted by the spectral fit to the CRIRES spectrum is consistent with both these hypotheses. The near-IR continuum itself is thought to be dominated by thermal emission of warm dust. Therefore, the location of the $\mathrm{CO}$ emitting region is consistent with the presence of a gaseous disc interior to the near-IR continuum emitting region (which has an inner radius of approximately $5 \mathrm{AU}$ ). This supports the association of the continuum emitting region with the inner rim of a dusty disc.

The most compelling support for the disc scenario is provided by the kinematic element of the modelling. The excellent agreement between the model spectral profile and the data proves that the kinematics of the emitting material are entirely consistent with Keplerian motion. Finally, we note that the AMBER differential phase signature provides a third argument in terms of photocentric displacement on scales of 0.15 mas. The observed signature is found to be fully consistent with the predictions of the best fitting model. The combination of high spatial and spectral precision provides stringent constraints on the kinematics of line emission regions. Therefore, the excellent agreement between the data and disc model in both the spatial and spectral domains is compelling evidence that the $\mathrm{CO}$ emission originates in a Keplerian disc.

As far as the nature of this disc is concerned, we observe the following. We note that the relatively low inner temperature of the disc suggests there is an absence of hot material close to the central star. Consequently, this is consistent with the presence of an inner gap (see Fig. 5). The derived inner radius of the $\mathrm{CO}$ emitting disc is $\sim 3 \mathrm{AU}$, which is larger than the (somewhat uncertain) binary separation of $a \sim 1.7$ AU (M2003). In other words, the model presented here confirms the suggestion of W12 that the HD 327083 system is surrounded by a circumbinary disc. The fact that the gaseous disc does not extend closer to the central star, where its inner rim temperature would be $\sim 5000 \mathrm{~K}$, provides further insight into the mass loss of this system. Specifically, the classification of HD 327083 as an interacting $\beta$ Lyrae type binary (M2003) can most likely be discounted, because of the absence of a circumstellar accretion disc. Also, the $\operatorname{sgB}[\mathrm{e}]$ model appears unfitting for this system (HD 327083 is listed as a $\operatorname{sgB}[\mathrm{e}]$ candidate by Kraus et al. 2009). The CO bandhead emission of such objects is expected to trace an equatorial outflow. Since we show that this model does not reproduce the data well, it appears unlikely that this object can be described by the standard $\mathrm{sgB}[\mathrm{e}]$ picture. This supports the hypothesis that the $\mathrm{B}[\mathrm{e}]$ behaviour of HD 327083 is related to its binarity.

This conclusion raises the question of whether there are any other systems similar to HD 327083. The CO emission contains two clues regarding the origin of the material surrounding HD 327083. First, we reiterate that the material is confined to a circumbinary disc. Secondly, we note that the gaseous circumbinary disc exhibits no evidence of variability. CO emission traces hot dense gas in the inner-most regions of discs and is sensitive to the surface density of the emitting region. Therefore, it is likely that the CO emission of HD 327083 would be sensitive to varying mass injection into the circumbinary disc. Consequently, the lack of variability suggests that the circumbinary disc is a relatively stable structure. 
Considering these points, the binary HD 327083 appears very similar to the binary A[e] star HD 62623 recently studied by Millour et al. (2011). HD 62623 is also a binary and Millour et al. (2011) demonstrate that a circumbinary disc surrounds the system. To explain the characteristics of this system, Plets et al. (1995) proposed that angular momentum transfer occurs through the L2 Lagrangian point of the system. As a result, mass lost from the primary supergiant by its radiatively driven wind is deposited into a stable dense circumbinary disc (which is consistent with the observations of Millour et al. 2011). If this scenario applies to HD 327083, the mass injection into the disc would be less dependent on orbital phase than the Roche Lobe overflow at periastron suggested by M2003. Therefore, this scenario could explain the lack of variability in the $\mathrm{CO}$ emission. Furthermore, this scenario also explains the presence of a circumbinary disc. Consequently, the environment of HD 327083 is entirely consistent with this scenario. As a result, HD 327083 could belong to the class of massive binaries which feature circumbinary discs thought to be due to the channeling of the primary's wind through the L 2 point of the system (currently exemplified by HD 62623 and possibly also $v$ Sgr, see Millour et al. 2011; Bonneau et al. 2011).

As a final remark, we note that this paper demonstrates the diagnostic potential of high spectral resolution observations of $\mathrm{CO}$ bandhead emission coupled with information on small angular scales as provided by VLTI observations. This approach offers a means to directly probe the circumstellar kinematics of $\mathrm{sgB}[\mathrm{e}]$ stars. Recent observations have found additional evidence for Keplerian structures around other sgB[e] stars, contrary to expectations based on the "dual outflow" sgB[e] model (Aret et al. 2012). Therefore, it is clear we have yet to arrive at a complete understanding of these objects. To systematically study the circumstellar environments of $\mathrm{sgB}[\mathrm{e}]$ stars, we have conducted a CRIRES survey of Galactic sgB[e] and $\mathrm{B}[\mathrm{e}]$ stars. The CRIRES observations presented here were drawn from this survey and demonstrate the quality and potential of the data. Analysis of the entire sample will be presented in a forthcoming paper (Muratore et al., in prep.) and follow-up VLTI observations are planned.

\section{Conclusion}

This paper presents high spectral resolution (25 and $6 \mathrm{~km} \mathrm{~s}^{-1}$ ) AMBER and CRIRES observations of the supergiant $\mathrm{B}[\mathrm{e}]$ star HD 327083. The observations spectrally resolve the CO bandhead emission of this object for the first time and provide new insights into its immediate environment. Here we list the key results.

- We find no evidence that the CO bandhead emission of HD 327083 is variable. Since the observations span the majority of the estimated period of the binary system, this suggests that the structure of the $\mathrm{CO}$ emitting region is not strongly dependent on orbital phase.

- The CRIRES observations of HD 327083's CO 1st overtone bandhead emission are fit to an excellent degree by a model of a Keplerian rotating disc.

- A differential phase signature corresponding to a photocentre displacement of $\sim 0.15$ mas is observed. This is well reproduced by the same model that fits the high spectral resolution CRIRES spectrum. The agreement between the model and observations in both the spatial and spectral domains is compelling evidence that the CO emission of HD 327083 originates in a Keplerian disc.
- The inner edge of the best fitting CO disc is approximately $3 \pm 0.3 \mathrm{AU}$. This places it interior to the previously detected continuum emitting disc (which has an inner radius of approximately $5 \mathrm{AU}$ ), but is sufficiently large for the $\mathrm{CO}$ emitting disc to be circumbinary.

- We show that a model of an equatorial outflow cannot reproduce the data as well as the Keplerian disc model. This suggests that the standard $\mathrm{sgB}[\mathrm{e}]$ scenario is not applicable to HD 327083, which supports the hypothesis that the $\mathrm{B}[\mathrm{e}]$ behaviour of HD 327083 is due to binarity.

Acknowledgements. We thank the anonymous referee for his/her constructive comments. H.E.W. acknowledges the financial support of the MPIfR in Bonn. J.D.I. is grateful for a studentship from the Science and Technology Facilities Council of the UK. A. Liermann is thanked for a careful reading of the manuscript. The NSO/Kitt Peak FTS data used here were produced by NSF/NOAO. This paper makes use of the high spectral resolution $K$-band library of standard spectra provided by the GEMINI Observatory, which is operated by the Association of Universities for Research in Astronomy, Inc., under a cooperative agreement with the NSF on behalf of the international Gemini partnership of Argentina, Australia, Brazil, Canada, Chile, the United Kingdom, and the United States of America. Finally, this research has made use of the AMBER data reduction package of the Jean-Marie Mariotti Center ${ }^{4}$.

\section{References}

Aret, A., Kraus, M., Muratore, M. F., \& Borges Fernandes, M. 2012, MNRAS, 423, 284

Berthoud, M. G., Keller, L. D., Herter, T. L., Richter, M. J., \& Whelan, D. G. 2007, ApJ, 660, 461

Blind, N., Boffin, H. M. J., Berger, J.-P., et al. 2011, A\&A, 536, A55

Bonneau, D., Chesneau, O., Mourard, D., et al. 2011, A\&A, 532, A148

Chelli, A., Utrera, O. H., \& Duvert, G. 2009, A\&A, 502, 705

Domiciano de Souza, A., Bendjoya, P., Niccolini, G., et al. 2011, A\&A, 525, A22 Fullerton, A. W. 1990, Ph.D. Thesis, Toronto Univ., Ontario

Kaeufl, H.-U., Ballester, P., Biereichel, P., et al. 2004, in SPIE Conf. Ser., 5492, 1218

Kraus, M., Krügel, E., Thum, C., \& Geballe, T. R. 2000, A\&A, 362, 158

Kraus, S., Hofmann, K., Malbet, F., et al. 2009, A\&A, 508, 787

Kraus, S., Calvet, N., Hartmann, L., et al. 2012, ApJ, 746, L2

Lachaume, R. 2003, A\&A, 400, 795

Lamers, H. J. G. L. M., Zickgraf, F.-J., de Winter, D., Houziaux, L., \& Zorec, J. 1998, A\&A, 340, 117

Machado, M. A. D., \& de Araújo, F. X. 2003, A\&A, 409, 665

Martin, S. C. 1997, ApJ, 478, L33

McGregor, P. J., Hyland, A. R., \& Hillier, D. J. 1988, ApJ, 324, 1071

Millour, F., Chesneau, O., Borges Fernandes, M., et al. 2009, A\&A, 507, 317

Millour, F., Meilland, A., Chesneau, O., et al. 2011, A\&A, 526, A107

Miroshnichenko, A. S., Levato, H., Bjorkman, K. S., \& Grosso, M. 2003, A\&A, 406, 673

Monnier, J. D., \& Millan-Gabet, R. 2002, ApJ, 579, 694

Monnier, J. D., Berger, J.-P., Millan-Gabet, R., et al. 2006, ApJ, 647, 444

Najita, J., Carr, J. S., Glassgold, A. E., Shu, F. H., \& Tokunaga, A. T. 1996, ApJ, 462, 919

Oudmaijer, R. D., \& Bakker, E. J. 1994, MNRAS, 271, 615

Pelupessy, I., Lamers, H. J. G. L. M., \& Vink, J. S. 2000, A\&A, 359, 695

Petrov, R. G., Malbet, F., Weigelt, G., et al. 2007, A\&A, 464, 1

Plets, H., Waelkens, C., \& Trams, N. R. 1995, A\&A, 293, 363

Porter, J. M. 2003, A\&A, 398, 631

Puls, J., Vink, J. S., \& Najarro, F. 2008, A\&ARv, 16, 209

Tatulli, E., Millour, F., Chelli, A., et al. 2007, A\&A, 464, 29

Tatulli, E., Malbet, F., Ménard, F., et al. 2008, A\&A, 489, 1151

Štefl, S., Rivinius, T., Carciofi, A. C., et al. 2009, A\&A, 504, 929

Vink, J. S. 2009, in Eta Carinae and the supernova imposters, eds. R. M. Humphreys, \& K. Davidson (Springer) [arXiv: 0905. 3338]

Wheelwright, H. E., Oudmaijer, R. D., de Wit, W. J., et al. 2010, MNRAS, 408, 1840

Wheelwright, H. E., Bjorkman, J. E., Oudmaijer, R. D., et al. 2012a, MNRAS, 423, L11

Wheelwright, H. E., de Wit, W. J., Oudmaijer, R. D., \& Vink, J. S. 2012b, A\&A, 538, A6

Zickgraf, F.-J. 2006, in Stars with the B[e] Phenomenon, eds. M. Kraus, \& A. S. Miroshnichenko (San Fransico, CA: ASP), ASP Conf. Ser., 355, 211 Zickgraf, F., Wolf, B., Stahl, O., Leitherer, C., \& Klare, G. 1985, A\&A, 143, 421

4 Available at http://www . jmmc . fr/amberdrs 